\title{
Ocular Involvement in Sjögren's Syndrome: Advances in Therapy
}

\author{
John A. Gonzales ${ }^{1,2, *}$ \\ Thomas M. Lietman ${ }^{1,2}$
}

\author{
Address \\ ${ }^{*}, 1$ Francis I. Proctor Foundation, University of California, 95 Kirkham Street, San \\ Francisco, CA, 94122, USA \\ Email: john.gonzales@ucsf.edu \\ ${ }^{2}$ Department of Ophthalmology, University of California, San Francisco, CA, USA
}

Published online: 8 February 2018

(C) The Author(s) 2018. This article is an open access publication

This article is part of the Topical Collection on Other CTD: Inflammatory

Myopathies and Sjogren's

Keywords Sjögren's syndrome · Keratoconjunctivitis sicca · Immunomodulatory therapy

\begin{abstract}
Purpose This article seeks to identify immunomodulatory therapy that holds promise for managing the ocular features of Sjögren's syndrome.

Recent findings There is an unmet need in therapies that can modulate the inflammatory damage that mediates keratoconjunctivitis sicca. While tear substitutes abound, there is a possibility that prevention of lacrimal gland and conjunctival inflammation may preserve tear production. Topical immunomodulatory therapy can improve some of the signs of keratoconjunctivitis sicca. Systemic immunomodulatory therapy appears to improve symptoms of ocular dryness, but improvement of signs of dry eye disease can be equivocal. Summary Topical immunomodulatory therapy can be effective in managing some of the signs of keratoconjunctivitis sicca. Systemic immunomodulatory therapy may play a role in managing or preventing the systemic inflammation that can lead to dry eye disease, but systemic therapy is currently employed when there are also systemic complications in Sjögren's syndrome. Future studies are needed to elucidate the utility of systemic immunomodulatory therapy in refractory cases of keratoconjunctivitis sicca.
\end{abstract}

\section{Introduction}

Henrik Sjögren (born in 1899 in Köping, Sweden) was perhaps destined to have his eponymous condition known to the world over. Indeed, in Swedish, sjö means "lake" and gre means "branch". The fact that Sjögren's syndrome is related to a reduced tear lake and that branches of the corneal basal nerve plexus can exhibit pathology is an interesting coincidence. Sjögren's interest in aqueous 
deficiency was perhaps sparked by the aqueous deficiency in the setting of trachoma harbored by the Ukrainian immigrants he was tasked with examining in his early career. In 1930, he described a case of a woman with rheumatism and hyposecretion of the lacrimal and salivary glands. It was this case where he introduced the new word keratoconjunctivitis sicca in the context of significant conjunctival and corneal staining with both rose bengal and methylene blue [1].

In 1933, Sjögren presented his doctoral thesis at the Karolinska Institute, which was entitled, "Kenntnis der Keratokonjunktivitis Sicca filiformisbeihypofunktion der Tranendriisen" and in which he described mostly arthritic women with keratoconjunctivitis sicca [2]. Clinical and pathological features were discussed and he made a point to differentiate keratoconjunctivitis sicca from xerophthalmia seen in vitamin A deficiency. How, indeed, are these two conditions differentiated from each other clinically? In keratoconjunctivitis sicca, there is reduced aqueous production (Sjögren confirmed this with Schirmer testing) $[2,3]$. In vitamin A deficiency, there is typically no aqueous deficiency, but rather a deficiency in mucin due to destruction of the goblet cells that produce mucin [4]. Sjögren postulated that his cases represented a systemic disease. Sadly, Sjögren's presentation did not receive the recognition it deserved and he was disqualified from receiving his $\mathrm{PhD}$. His academic career was effectively over. However, despite this setback, Sjögren was very much alive to see the systemic condition that he described so well (and posited a systemic dysfunction) named for him. Sjögren died in 1986 and he was intimately involved with the major descriptions and characterizations of the disease. Sjögren was also made professor at Gothenberg University and was made an honorary member of the Swedish Rheumatological Society.

\section{Ocular manifestations of Sjögren's syndrome}

As Sjögren described, the aqueous deficiency leads to keratoconjunctivitis sicca with dryness of the cornea and conjunctiva. The lacrimal gland can occasionally be enlarged, but typically in early stages, the lacrimal gland dysfunction leads to decreased aqueous (tear) production. However, there are exocrine glands in the conjunctiva and these are also affected in Sjögren's syndrome as well. Often, the cornea can manifest features of a reduced tear film in which the corneal epithelium may demonstrate epithelial erosions initially. Later, corneal filaments that are extremely painful can develop. In later stages of the disease when there is profound aqueous deficiency, the conjunctiva loses its lustrous and wet appearance and may easily stain with the same fluorescein dye that is best reserved for evaluating the cornea. Early involvement of conjunctival desiccation is best demonstrated with concentrated lissamine green $1 \%$. Instilling the drop onto the superior bulbar conjunctiva allows the lissamine green drop to adequately stain both the superior and inferior bulbar conjunctivae. In some cases, the inflammatory component may manifest as superior limbic keratoconjunctivitis [5].

The lacrimal gland reveals both focal and diffuse lymphocytic infiltrations on histology. Occasionally, there can be proliferation of the terminal ducts within the lacrimal gland leading to epimyoepithelial islands within zone of lymphoid hyperplasia [6]. In the histopathology of the conjunctival stroma, there is a lymphocytic infiltration and a reduction in the number of conjunctival goblet cells.

The ophthalmologist assesses keratoconjunctivitis sicca by evaluating aqueous production by the lacrimal gland. 
Inserting filter paper strips in the corner of each eye and quantifying how far the tears extend down the filter paper (in millimeters) over 5 min allows the ophthalmologist to quantify how much tears the lacrimal gland produces. Values below $5 \mathrm{~mm}$ are considered low, but this test exhibits variability.

Tear break up time

Instilling fluorescein dye into the eye allows for quantification of the evaporation of tears (in seconds) from the cornea after blinking. Tears that evaporate in less than $10 \mathrm{~s}$ after blinking are considered abnormal.

Corneal staining

Fluorescein dye also allows for evaluating the corneal staining pattern. Punctate epithelial erosions and filaments are more easily seen with fluorescein dye.

Conjunctival staining

Advanced keratoconjunctivitis sicca with profoundly dry conjunctiva can be appreciated with fluorescein dye, but the vital dyes lissamine green and rose bengal show conjunctival epitheliopathy in earlier, less advanced stages of dry eye disease. Lissamine green does not carry the discomfort that rose bengal does, so it is more typically used in clinic. We use concentrated lissamine green $1 \%$ (compounded) as opposed to lissamine green-impregnated strips that are wet with saline or sterile water, though many centers use the strips as they are commercially available [7].

Questionnaires for dry eye symptoms

There are a variety of tools to assess dry eye symptoms including the Ocular Surface Disease Index as well as visual analog scales [8-10]. Symptoms of ocular dryness and discomfort are important to assess in Sjögren's syndrome partly because patient-reported symptoms of dry eye often do not correlate with signs of keratoconjunctivitis sicca $[11 \bullet \bullet, 12,13 \bullet \bullet]$.

\section{General initial approach}

Topical therapy to replenish the ocular surface's hydration in the form of artificial tears and ointments is outside of the focus for this article. An excellent review of the management of dry eye disease in Sjögren's syndrome using topical therapies has been presented elsewhere [14•]. Briefly, there is a multitude of artificial tear therapies available over the counter to patients. Most ophthalmologists recommend to their patients using preservative-free artificial tears, particularly if lubrication is being used more frequently than four times daily. Additionally, serum tears (made from the patient's own serum drawn by phlebotomy) can provide additional comfort. Because there is a reduction in goblet cells and mucin production in the conjunctiva, mucin secretagogues (including diquafosol and rebamipide) may help with ocular surface lubrication [15]. Topical mucin secretagogues are not available in the USA, however. In severe or refractory cases of keratoconjunctivitis sicca, scleral lenses may provide improvement in comfort, vision, and signs of keratoconjunctivitis sicca. Scleral 
lenses are rigid lenses that rest on the sclera and are vaulted off the cornea. This creates a reservoir in which fluid can be placed and, when the patient places on their eye, allows for the cornea to be bathed in the lubricating fluid. An intranasal tear neurostimulator, which functions by an element that is placed on the nasal cavity's mucosa and is then controlled by a rechargeable stimulator unit, can help in increasing lacrimal gland tear production [16].

\section{Topical immunomodulatory therapy}

Because inflammatory mediators are present on the ocular surface, the use of medications that abrogate or modulate the inflammatory response can play a role in decreasing inflammatory-mediated signs and symptoms of keratoconjunctivitis sicca. If we consider that non-infectious intraocular inflammation (uveitis) is managed by using therapies that target the inflammatory response, it is reasonable to consider that inflammation on the ocular surface could be managed using a similar approach.

Corticosteroids have myriad effects on inflammatory pathways and are first-line agents in the management of ocular inflammation including scleritis, keratitis, and uveitis [17]. In the setting of dry eye disease, topical corticosteroids have been shown to decrease corneal fluorescein staining, improve tear break up time, and improve Schirmer 1 testing $[18,19]$. A recurrence of signs of keratoconjunctivitis sicca may recur after stopping topical corticosteroids [19]. Lowpotency topical corticosteroid treatment with fluoromethalone (FML) in conjunction with artificial tears when compared to artificial tears alone and combination artificial tears and flurbiprofen (a topical nonsteroidal) has been shown to not only improve fluorescein and rose bengal staining but also demonstrate higher numbers of goblet cells on impression cytology in a randomized trial (involving Sjögren's syndrome and non-Sjögren's participants) [20]. Schirmer 1 testing, however, did not improve.

Long-term use of topical corticosteroids is typically not recommended. The use of topical corticosteroids carries risk of elevated intraocular pressure, which can lead to glaucoma, or the generation of a posterior subcapsular cataract. However, low-frequency (i.e., twice daily or less) topical corticosteroids (such as prednisolone acetate 1\%) are used long-term in other ocular conditions including herpetic interstitial keratitis and keratouveitis, and non-infectious uveitis [21,22]. Indeed, in our clinical practice (as we have experienced with using maintenance doses of topical corticosteroids for many years and decades), we find that the side effects of topical corticosteroids can be monitored provided patients return for regular visits to check intraocular pressure and lens status.

Our preferences for steroid drops in the setting of keratoconjunctivitis sicca are for a solution-based drop, which include (in order of decreasing potency) dexamethasone $0.1 \%$, prednisolone sodium phosphate $1 \%$, and methylprednisolone $1 \%$. Non-preserved formulations of these topical corticosteroids can be produced by a compounding pharmacy. Since prednisolone acetate $1 \%$ is a suspension, the prednisolone particles may cause more irritation on the ocular 
surface of those experiencing ocular discomfort, including those with keratoconjunctivitis sicca. Moreover, particle sizes in prednisolone acetate vary depending on manufacturer [23]. We feel that since solutions lack the particulates, there may be less ocular discomfort experienced by the patient.

The calcineurin (or T cell) inhibitor, cyclosporine, has been FDA approved for dry eye (Restasis, cyclosporine ophthalmic $0.05 \%$, Allergan, Inc.). Cyclosporine can also be compounded into higher concentrations such as $0.1,0.2$, and $0.4 \%$. Cyclosporine $0.05 \%$ and $0.1 \%$ have been shown to improve corneal fluorescein staining and Schirmer with anesthesia testing more than placebo (vehicle) in randomized trials [24]. However, it is important to note that both cyclosporine and placebo groups showed an improvement with therapy at some time points (regardless of randomization). Additionally, all arms showed an improvement in both conjunctival staining and Schirmer 1 (without anesthetic) testing, and there was no statistically significant difference between the arms with respect to these parameters. The vehicle used in the study (which was the same as the emulsive agent used in the cyclosporine emulsions) was a proprietary formulation of non-preserved castor oil in water [24]. The improvements in ocular features of keratoconjunctivitis sicca, then, may have been related to the formulation of the vehicle. Additionally, patients in this Allergan-funded trial received preservative-free artificial tears (REFRESH Lubricant Eye Drops, Allergan, Inc.) to be used "as frequently as needed" [24]. Topical cyclosporine $0.05 \%$ has been shown to reduce lymphocyte activation markers in the conjunctiva of those with keratoconjunctivitis sicca (in both Sjögren's syndrome and non-Sjögren's syndrome), but not the conjunctival T cell infiltrate when compared to the vehicle (Allergan's proprietary vehicle) [25].

Lifitegrast (Xiidra, Shire, USA) was recently approved by the US FDA for the treatment of dry eye. It inhibits lymphocyte function-associated antigen 1 (LFA-1), which is a member of the integrin family, from binding to intercellular adhesion molecule 1 (ICAM-1). T cells that mediate inflammation express LFA-1, so inhibiting the interaction between LFA-1 and ICAM-1 leads to the prevention of T cells from becoming activated as well as migrating to target tissues.

Phase 3 trials comparing lifitegrast to placebo have recently occurred. In one randomized phase 3 trial, after a 2-week placebo run-in, participants were randomized to receive lifitegrast or placebo in the study eye. At 12 weeks, primary endpoints included change from baseline to 12 weeks, from baseline in eye dryness as measured on the visual analog scale as well as ocular signs of keratoconjunctivitis sicca (inferior corneal fluorescein staining in the study eye). While there was a statistically significant greater improvement in patientreported symptoms in eye dryness in the lifitegrast group compared to placebo, there was no difference between the two arms with respect to inferior corneal staining with fluorescein [26].

In another randomized phase 3 trial in which participants were randomized to lifitegrast or placebo, eye dryness score on a visual analogue scale improved to a greater degree in the lifitegrast arm 
compared to placebo. However, ocular discomfort (distinct from dryness) did not differ between arms. While there was a trend for a greater proportion of participants in the lifitegrast arm compared to placebo experiencing an improvement in secondary outcome measures of signs of keratoconjunctivitis sicca (conjunctival redness score, corneal fluorescein staining, conjunctival lissamine green staining, and Schirmer 1 testing), these were not statistically significant $[27 \bullet \bullet]$.

\section{Systemic immunomodulatory and biologic therapy}

The treatment of keratoconjunctivitis sicca with systemic immunosuppression merits investigation. Given that the conjunctiva and lacrimal gland ductules exhibit an inflammatory infiltrate on histopathology [6, 28, 29], it bears at least some consideration to use systemic immunosuppression to prevent inflammatory changes in the lacrimal gland and conjunctiva, thereby preserving function of the exocrine glands they contain.

Turning to intraocular inflammation (uveitis) where such an approach is standard may serve as a potential model for an approach to some types of keratoconjunctivitis sicca, particularly in Sjögren's syndrome [17]. Indeed, systemic therapy has been associated with improvement in the ocular signs and symptoms of Sjögren's syndrome as presented below. Insight into the efficacy of systemic immunomodulatory medication decreasing conjunctival anatomical changes associated with Sjögren's syndrome has been provided by one group performing tarsal conjunctival biopsies before and after starting systemic immunosuppression. In their two cases (both patients had Sjögren's syndrome in the setting of rheumatoid arthritis), conjunctival biopsy prior to starting immunosuppression revealed decrease in height of microvilli and lack of branching microvilli on electron microscopy. Following the institution of systemic antimetabolite therapy (one patient received azathioprine while the other received methotrexate), repeat tarsal conjunctival biopsy demonstrated an increase in number, height, and branching of the microvilli [30].

While recommendations exist for employing systemic immunomodulatory medications (particularly hydroxychloroquine and methotrexate) in Sjögren's syndrome, such recommendations are typically for widespread systemic involvement (inflammatory musculoskeletal pain) [31 $\left.{ }^{\circ}\right]$. The anti-tumor necrosis factor inhibitor biologics have previously not been recommended for Sjögren's syndrome unless there is an associated systemic autoinflammatory condition that requires the use of such therapy (rheumatoid arthritis, for example). There has been consideration, however, for the biologic rituximab in Sjögren's disease, including when there is keratoconjunctivitis sicca, though the recommendations for this indication are weak. Stronger recommendations for rituximab's use are for cryoglobulinemia, vasculitis, inflammatory arthritis, and peripheral neuropathies in the setting of Sjögren's syndrome $\left[31^{\circ}\right]$. Nevertheless, the interest in immunomodulatory and biologic therapy for Sjögren's syndrome is increasing and we will likely see more clinical trials designed to evaluate the efficacy of such therapeutics in keratoconjunctivitis sicca. 


\section{Hydroxychloroquine and chloroquine}

Chloroquine and hydroxychloroquine are antimalarial medications that also have anti-inflammatory properties and have been used extensively in rheumatoid arthritis and systemic lupus erythematosus.

In a study of Sjögren's syndrome patients that had been taking hydroxychloroquine for at least 2 years, all 32 patients were discontinued from their antimalarial for 3 months. There was a statistically significant worsening of tear break up time, Schirmer 1 scores, and corneal and conjunctival staining [32]. Additionally, patients became symptomatic with respect to ocular sensations including gritty and burning sensations. In a retrospective, openlabel study of patients with primary Sjögren's syndrome taking hydroxychloroquine for at least 2 years, there was an improvement in ocular pain and dryness as well as features of keratoconjunctivitis sicca (corneal staining and Schirmer 1 testing) [33]. More recently, in a masked, randomized trial comparing hydroxychloroquine to placebo in primary Sjögren's syndrome, there was no change in either group between baseline and week 12 with respect

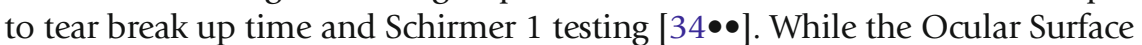
Disease Index (OSDI) improved for both groups at weeks 6 and 12, there was no statistically significant difference between the two arms.

Because hydroxychloroquine can cause retinal toxicity (specifically a maculopathy), baseline testing including macula optical coherence tomography, central visual field testing, and fundus autofluorescence should be performed at baseline and at follow-up intervals.

\section{Antimetabolites}

\section{Methotrexate}

Synthesized in the 1940s, methotrexate found use in the 1950s for psoriasis and then in the 1960s for rheumatoid arthritis [35-37]. By targeting mammalian dihydrofolate reductase (an enzyme important for purine and thymidylate synthesis), methotrexate abrogates the growth and division of rapidly dividing cells, which in the case of autoinflammatory conditions includes inflammatory cells. Methotrexate is a mainstay for steroid-sparing control of ocular inflammation, including uveitis and scleritis [38]. In a small case series, two patients with intractable keratoconjunctivitis sicca (one patient with Sjögren's syndrome and the other with rheumatoid arthritis and, thus, perhaps secondary Sjögren's syndrome) responded well to methotrexate alone or methotrexate used in combination with a biologic [39]. While methotrexate has well-documented efficacy in ocular inflammatory conditions like uveitis and scleritis, there is scant data in the literature that would support its use specifically to improve keratoconjunctivitis sicca. Future studies utilizing methotrexate for Sjögren's syndrome-related keratoconjunctivitis sicca are needed.

\section{Mycophenolate mofetil}

Mycophenolate mofetil, which inhibits inosine monophosphate dehydrogenase, blocks de novo purine synthesis, leading to a decrease in DNA synthesis in 
proliferating lymphocytes. Mycophenolate mofetil, like methotrexate, plays an important role as a first-line steroid-sparing agent for ocular inflammation [40].

In one open-label study, patients with primary Sjögren's syndrome were treated with mycophenolate sodium. Most patients reported a subjective improvement in their dry eyes by the visual analog scale. There was, however, no improvement in signs of keratoconjunctivitis sicca. Мycophenolate did lead to improvement in hypergammaglobulinemia and reduction in serologic rheumatoid factor in some patients [41]. In a small retrospective study, patients with sensory neuropathies (including paresthesias and pain) showed an improvement in their handicap scores, but ocular pain specifically was not assessed [42]. Similar to methotrexate, future studies using mycophenolate mofetil for Sjögren's syndrome are needed to demonstrate efficacy that is reasonable in light of medication side effects.

\section{Biologics}

\section{Rituximab}

Rituximab is a chimeric monoclonal antibody that targets the B-

lymphocyte surface antigen, CD20, and has been effective in the control of ocular inflammation, particularly in scleritis and ocular cicatricial pemphigoid [43-45]. In the setting of Sjögren's syndrome, two small, masked trials where participants were randomized to placebo or rituximab showed that symptoms of dryness (including ocular) improved with rituximab $[46,47 \bullet \bullet]$. However, a larger multicenter randomized trial, placebo-controlled trial, showed that rituximab did not improve Schirmer 1 testing, tear break up time, or ocular symptoms [48].

\section{Belimumab}

As an inhibitor B cell-activating factor (BAFF), the monoclonal antibody belimumab may decrease B cell-mediated inflammation in exocrine glands. While an open-label phase II study showed that Schirmer testing did not change with therapy, symptoms of dryness (including ocular) improved [49]. Currently, in the USA, belimumab is approved by the US Food and Drug Administration (FDA) for systemic lupus erythematosus.

\footnotetext{
Abatacept
}

Abatacept has demonstrated efficacy in reducing disease activity in Sjögren's syndrome. While some studies have shown no statistically significant change in tear break up time with therapy [50], other studies involving Sjögren's syndrome in the setting of rheumatoid arthritis have shown an improvement in Schirmer testing [ $[51 \bullet]$, which is felt to be a better indicator of lacrimal gland function $[7,52 \bullet]$.

There is an unmet need for disease-modifying therapy in Sjögren's syndrome. First-line therapy for managing keratoconjunctivitis sicca in Sjögren's syndrome 
typically involves using lubricating eye drops (including artificial or serum tears), though scleral lenses can offer significant improvement. These therapies, however, do not modulate the inflammatory processes in the lacrimal gland and conjunctiva that can lead to the discomfort, pain, and reduced vision in keratoconjunctivitis sicca. Specific topical therapy may address this at a local level and there is a wide variety of topical immunomodulators to choose from: corticosteroids, cyclosporine, and lifitegrast. However, given that there is a systemic drive for the inflammation occurring on the ocular surface, the use of systemic immunosuppression is intriguing because there is a chance to induce a remission with such an approach. Moreover, there is a potential for the systemic complications associated with Sjögren's syndrome to be treated or perhaps prevented altogether when keratoconjunctivitis sicca in Sjögren's syndrome is present. Future randomized trials in which immunomodulatory or biologic therapy is instituted for ocular involvement in Sjögren's syndrome are needed.

\section{Compliance with Ethical Standards}

\section{Conflict of Interest}

John A. Gonzales declares that he has no conflict of interest.

Thomas M. Lietman declares that he has no conflict of interest.

\section{Human and Animal Rights and Informed Consent}

This article does not contain any studies with human or animal subjects performed by any of the authors.

Open Access This article is distributed under the terms of the Creative Commons Attribution 4.0 International License (http://creativecommons.org/licenses/by/4.0/), which permits unrestricted use, distribution, and reproduction in any medium, provided you give appropriate credit to the original author(s) and the source, provide a link to the Creative Commons license, and indicate if changes were made.

\section{References and Recommended Reading}

Papers of particular interest, published recently, have been

highlighted as:

- Of importance

$\bullet$ Of major importance

1. Sjögren, H. Keratoconjunctivitis sicca. Hygiea, 829 (1930).

2. Sjögren, H. ZurKenntnis der Keratoconjunctivitis sicca. Keratitis filiformisbeiHypofunktion der Tränendrüsen. Actaophthalmologica, 1-151 (1933).

3. Sjögren, H. Keratconjunctivitis sicca. (in Tran Ophthalmol Section Swedish Med Assoc. 1929-1931). Actaophthalmologica10, 403-409 (1932).

4. Lin, P., Fintelmann, R.E., Khalifa, Y.M., Bailony, M.R. \&Jeng, B.H. Ocular surface disease secondary to vitamin A deficiency in the developed world: it still exists.
Archives of ophthalmology (Chicago, Ill. : 1960)129, 798799 (2011).

5. Thygeson P, Kimura SJ. Chronic conjunctivitis. Trans Am Acad Ophthalmol Otoloaryngol 1963;67(4):494517.

6. Font RL, Yanoff M, Zimmerman LE. Benign lymphoepithelial lesion of the lacrimal gland and its relationship to Sjogren's syndrome. Am J Clin Pathol. 1967;48:365-76.

7. Whitcher JP, et al. A simplified quantitative method for assessing keratoconjunctivitis sicca from the Sjogren's 
Syndrome International Registry. Am J Ophthalmol. 2010;149:405-15.

8. Gabbriellini G, et al. Ocular Surface Disease Index (OSDI): a potential useful instrument for the assessment of vision-targeted health-related quality of life (VT-HRQ) in primary Sjogren's syndrome (pSS) clinical trials? Clin Exp Rheumatol. 2012;30:812-3.

9. Schiffman RM, Christianson MD, Jacobsen G, Hirsch JD, Reis BL. Reliability and validity of the Ocular Surface Disease Index. Arch Ophthalmol (Chicago, Ill. : 1960). (2000);118:615-21.

10. Vitale S, Goodman LA, Reed GF, Smith JA. Comparison of the NEI-VFQ and OSDI questionnaires in patients with Sjogren's syndrome-related dry eye. Health and Quality of life outcomes. (2004);2:44.

11.• Adatia FA, et al. Correlation between corneal sensitivity, subjective dry eye symptoms and corneal staining in Sjogren's syndrome. Can J Ophthalmol J Can'Ophtalmol. (2004);39:767-71.

This article discusses the lack of correlation between signs and symptoms of dry eye

12. Begley CG, et al. The relationship between habitual patient-reported symptoms and clinical signs among patients with dry eye of varying severity. Invest Ophthalmol Vis Sci. 2003;44:4753-61.

13.• Whitcher JP Jr, Gritz DC, Daniels TE. The dry eye: a diagnostic dilemma. Int Ophthalmol Clin. 1998;38:23-37.

This article discusses the lack of correlation between signs and symptoms of dry eye

14.• Foulks GN, et al. Clinical guidelines for management of dry eye associated with Sjogren disease. Ocular Surf. 2015;13:118-32.

Excellent review of topical ocular therapy in Sjögren's syndrome

15. Caffery B, et al. MUC1 expression in Sjogren's syndrome, KCS, and control subjects. Mol Vis. 2010;16:1720-7.

16. Friedman NJ, et al. A nonrandomized, open-label study to evaluate the effect of nasal stimulation on tear production in subjects with dry eye disease. Clin Ophthalmol (Auckland, NZ). 2016;10:795-804.

17. Jabs DA, et al. Guidelines for the use of immunosuppressive drugs in patients with ocular inflammatory disorders: recommendations of an expert panel. Am J Ophthalmol. 2000;130:492-513.

18. Marsh P, Pflugfelder SC. Topical nonpreserved methylprednisolone therapy for keratoconjunctivitis sicca in Sjogren syndrome. Ophthalmology. 1999;106:811-6.

19. Hong S, Kim T, Chung SH, Kim EK, Seo KY. Recurrence after topical nonpreserved methylprednisolone therapy for keratoconjunctivitis sicca in Sjogren's syndrome. J Ocul Pharmacol Ther: Off J Assoc Ocul Pharmacol Ther. 2007;23:78-82.

20. Avunduk AM, Avunduk MC, Varnell ED, Kaufman HE. The comparison of efficacies of topical corticosteroids and nonsteroidal anti-inflammatory drops on dry eye patients: a clinical and immunocytochemical study. Am J Ophthalmol. 2003;136:593-602.

21. Thorne JE, Woreta FA, Dunn JP, Jabs DA. Risk of cataract development among children with juvenile idiopathic arthritis-related uveitis treated with topical corticosteroids. Ophthalmology. 2010;117:1436-41.

22. Barron BA, et al. Herpetic Eye Disease Study. A controlled trial of oral acyclovir for herpes simplex stromal keratitis. Ophthalmology. 1994;101:1871-82.

23. Roberts CW, Nelson PL. Comparative analysis of prednisolone acetate suspensions. J Ocular Pharmacol Ther: Off J Assoc Ocular Pharmacol Ther. 2007;23:182-7.

24. Sall K, Stevenson OD, Mundorf TK, Reis BL. Two multicenter, randomized studies of the efficacy and safety of cyclosporine ophthalmic emulsion in moderate to severe dry eye disease. CsA Phase 3 Study Group. Ophthalmology. 2000;107:631-9.

25. Kunert KS, Tisdale AS, Stern ME, Smith JA, Gipson IK. Analysis of topical cyclosporine treatment of patients with dry eye syndrome: effect on conjunctival lymphocytes. Arch Ophthalmol (Chicago, Ill. : 1960). 2000;118:1489-96.

26. Tauber J, et al. Lifitegrast ophthalmic solution 5.0\% versus placebo for treatment of dry eye disease: results of the randomized phase III OPUS-2 study. Ophthalmology. 2015;122:2423-31.

27.••Holland, E.J., Luchs J., Karpecki P.M., Nichols K.K., Jackson M.A., Sall K., Tauber J., Roy M., Raychaudhuri A., Shojaei A. Lifitegrast for the treatment of dry eye disease: results of a phase III, randomized, double-masked, placebo-controlled trial (OPUS-3). Ophthalmology 124, 53-60 (2017), 1, https://doi.org/10.1016/j. ophtha.2016.09.025.

This reference involves a recent trial concerning the use of the newest topical anti-inflammatory therapy for dry eye disease, lifitegrast.

28. Belfort R Jr, Mendes NF. Identification of T and B lymphocytes in the human conjunctiva and lacrimal gland in ocular diseases. Brit J Ophthalmol. 1980;64:217-9.

29. Meyer E, et al. Light and electron microscopical study of the conjunctiva in sicca syndrome.

Ophthalmologica. J Int d'Ophtalmol. Int J Ophthalmol Zeitschrift fur Augenheilkunde. 1985; 190:45-51.

30. Scharf JM, Meyer E, Scharf JA, Nahir M, Zonis S. Effects of immunosuppressive drugs on the morphology of the microvilli in Sjogren's syndrome. Scand J Rheumatol. 1986;61(Supplement):215-9.

31.• Vivino FB, Carsons SE, Foulks G, Daniels TE, Parke A, Brennan MT, et al. New treatment guidelines for Sjogren's disease. Rheum Dis Clin N Am. 2016;42(3):531-51. https://doi.org/10.1016/j.rdc. 2016.03.010.

General treatment guidelines for Sjögren's syndrome are presented in this reference. This review also highlights the significant effect that Sjögren's syndrome has on quality of life. 
32. Yavuz S, Asfuroglu E, Bicakcigil M, Toker E. Hydroxychloroquine improves dry eye symptoms of patients with primary Sjogren's syndrome. Rheumatol Int. 2011;31:1045-9.

33. Fox RI, Dixon R, Guarrasi V, Krubel S. Treatment of primary Sjogren's syndrome with hydroxychloroquine: a retrospective, open-label study. Lupus. 1996;5(Suppl 1):S31-6.

34.• Yoon, C.H., Lee H.J., Lee E.Y., Lee E.B., Lee W.W., Kim M.K., Wee W.R. Effect of hydroxychloroquine treatment on dry eyes in subjects with primary Sjogren's syndrome: a double-blind randomized control study. J Korean Med Sci 31, 1127-1135 (2016), 7, https://doi. org/10.3346/jkms.2016.31.7.1127.

While this reference showed that there was no statistically significant difference in the Ocular Surface Disease Index (which relies heavily on patient-reported symptoms) or dry eye parameters including tear break up time, Schirmer, and corneal fluorescein staining, this was a well-designed (albeit with a small number of participants enrolled) double-masked randomized trial. This study demonstrates the need for randomized trials to specifically address ocular signs and patientreported symptoms of dry eyes in the setting of Sjögren's syndrome.

35. Cress RH, Deaver NL. Methotrexate in the management of severe psoriasis and arthritis: report of a case. South Med J. 1964;57:1088-90.

36. Enderlin M. Experiences with antimetabolite therapy of malignant forms of progressive chronic polyarthritis. Helvetica medicaacta. 1966;46(Supplementum):171.

37. Fosdick WM. Cytotoxic therapy in rheumatoid arthritis. Medi Clin North Am. 1968;52:747-57.

38. Gangaputra $S$, et al. Methotrexate for ocular inflammatory diseases. 2009; Ophthalmology, 116:21882198.e2181.

39. Cordero-Coma M, Anzaar F, Sobrin L, Foster CS. Systemic immunomodulatory therapy in severe dry eye secondary to inflammation. Ocular Immunol Inflamm. 2007;15:99-104.

40. Thorne JE, et al. Mycophenolate mofetil therapy for inflammatory eye disease. Ophthalmology. 2005; 112:1472-7.

41. Willeke P, et al. Mycophenolate sodium treatment in patients with primary Sjogren syndrome: a pilot trial. Arthritis Res Ther. 2007;9:R115.

42. Pereira PR, et al. Sjogren sensory neuronopathy (Sjogren ganglionopathy): long-term outcome and treatment response in a series of 13 cases. Medicine. 2016;95:e3632.

43. Suhler EB, et al. Rituximab therapy for refractory scleritis: results of a phase I/II dose-ranging, randomized, clinical trial. Ophthalmology. 2014;121:1885-91.

44. Cao JH, Oray M, Cocho L, Foster CS. Rituximab in the treatment of refractory noninfectious scleritis. Am J Ophthalmol. 2016;164:22-8.
45. You C, et al. Rituximab in the treatment of ocular cicatricial pemphigoid: a retrospective cohort study. Graefe's archive for clinical and experimental ophthalmology $=$ Albrecht von GraefesArchiv fur klinische und experimentelleOphthalmologie. 2017;255:1221-8.

46. Dass $\mathrm{S}$, et al. Reduction of fatigue in Sjogren syndrome with rituximab: results of a randomised, double-blind, placebo-controlled pilot study. Ann Rheum Dis. 2008;67:1541-4.

47.• Meijer, J.M., et al. Effectiveness of rituximab treatment in primary Sjogren's syndrome: a randomized, doubleblind, placebo-controlled trial. Arthritis and rheumatism62, 960-968 (2010).

Lissamine green staining of the conjunctiva showed improvement in this study utilizing biological therapy for Sjögren's syndrome.

48. Devauchelle-Pensec $\mathrm{V}$, et al. Treatment of primary Sjogren syndrome with rituximab: a randomized trial. Ann Int Med. 2014;160:233-42.

49. Mariette X, et al. Efficacy and safety of belimumab in primary Sjogren's syndrome: results of the BELISS open-label phase II study. Ann Rheum Dis. 2015;74:526-31.

50. Meiners PM, et al. Abatacept treatment reduces disease activity in early primary Sjogren's syndrome (openlabel proof of concept ASAP study). Ann Rheum Dis. 2014;73:1393-6.

51. Tsuboi H, et al. Efficacy and safety of abatacept for patients with Sjogren's syndrome associated with rheumatoid arthritis: Rheumatoid Arthritis with Orencia Trial toward Sjogren's syndrome Endocrinopathy (ROSE) trial-an open-label, oneyear, prospective study-interim analysis of 32 patients for 24 weeks. Modern rheumatol/Jpn Rheum Assoc. 2015;25:187-93.

The efficacy of abatacept in secondary Sjögren's syndrome is presented here.

52.• Tsuboi, H, Matsumoto I, Hagiwara S, Hirota T, Takahashi H, Ebe H, Yokosawa M, Yagishita M, Takahashi H, Kurata I, Ohyama A, Honda F, Asashima H, Miki H, Umeda N, Kondo Y, Hirata S, Saito K, Tanaka Y., Horai Y, Nakamura H, Kawakami A, Sumida T Effectiveness of abatacept for patients with Sjogren's syndrome associated with rheumatoid arthritis. An open label, multicenter, one-year, prospective study: ROSE (Rheumatoid Arthritis with Orencia Trial toward Sjogren's syndrome Endocrinopathy) trial. Modern Rheumatol/Jpn RheumAssoc 2016;26:891-899, 6, https://doi.org/10.3109/14397595.2016.1158773. In this reference, an open-label trial using abatacept for secondary Sjögren's syndrome showed a statistically significant improvement in Schirmer testing. 